\title{
A Portable Mirror Stand for Clinical Facial Photo Documentation
}

\author{
Laureen Supit, Theddeus OH Prasetyono
}

Division of Plastic Surgery, Department of Surgery, Faculty of Medicine, University of Indonesia and Cipto Mangunkusumo Hospital, Jakarta, Indonesia

In plastic surgery, patient photography is a vital component of clinical, educational, legal, and research documentation. Optimal acquisition of photographic data requires a dedicated photography studio or a three-dimensional anatomic scanner, both of which are financially impractical for most clinicians. Simplified photo standardization is proposed for use in random clinical settings by using a portable device called the Mirror Stand (MirS). This model device aims to mimic a studio environment by incorporating the basic elements of producing consistent photographs. The pilot MirS is designed for facial photography. Images of 40 random subjects were obtained using the MirS with three different cameras. Real anthropometric measurements of each subject were collected, compared with the photographic measurements, and analyzed. In this study, all three cameras produced equally reliable measurements. Actual facial measurements were comparable to the photogrammetric measurements obtained from photographs taken using the MirS. A constant formula was derived; it allowed the conversion of photographic values into real anthropometric values. The MirS produced consistent photographs with respect to the measurements. The photographs obtained could be translated reliably into their real anthropometric measurements. Therefore, the MirS can be applied in daily practice, providing an efficient alternative for obtaining a standard justifiable photograph.

Keywords Anthropometry / Cephalometry / Photogrammetry / Surgery, plastic

\author{
Correspondence: \\ Theddeus $\mathrm{OH}$ Prasetyono \\ Division of Plastic Surgery, \\ Department of Surgery, Faculty of \\ Medicine University of Indonesia and \\ Cipto Mangunkusumo Hospital, \\ Medical Staff Building 4th floor Jl. \\ Diponegoro, 71 Jakarta 13410, \\ Indonesia \\ Tel: $+62-817858899$ \\ Fax: +62-21-3905556 \\ E-mail: teddyohprasetyono@yahoo.com
}

\begin{abstract}
The authors would like to thank Nila Kesuma, MD, for her contribution in the construction of the MirS prototype; and Enjeline Hanafi, MD, for preparing the initial manuscript.
\end{abstract}

No potential conflict of interest relevant to this article was reported.

\section{INTRODUCTION}

The use of photography for medical documentation is vital, particularly for plastic surgeons. Its role as part of clinical, communication, educational, legal, and research aid is integral. Nonstandardized documentation leads to visual misinterpretation, diminishing the clinical value of photographs $[1,2]$. Numerous photography methodologies have been proposed to create specific conditions in clinical settings; these include specific patient positioning, certain placements of subjects and cameras, setting of camera angles, use of preset lighting techniques, and the use of specific cameras, which requires setting up a studio or studiolike environment [1,3-7]. A three-dimensional topographic scanner is also available but is quite costly.

In this paper, a portable and cost-effective method of standardized photodocumentation is proposed, which incorporates photogrammetry where real anthropometric measurements can be inferred from photographs [8]. A model device called the Mirror Stand (MirS) aims to mimic a studio environment by incorporating the basic elements of producing consistent unbiased 
photographs. The mirror system is adapted from a design by Kuhnel and Wolf [9] of Germany. The MirS prototype captures only the facial area, and any camera may be used to obtain the photographs. In this study, we have evaluated the reliability and reproducibility of the MirS in documenting clinical photographs of the face in healthy adult individuals along with its applicability to photogrammetric translation.

\section{IDEA}

This cross-sectional non-interventional study analyzed 40 healthy adult subjects selected by convenience sampling. Three digital cameras with varying lenses and megapixels were used in this study: Nikon Coolpix P300 (Tokyo, Japan), Olympus SP 800 UZ (Tokyo, Japan), and Canon EOS 600 D (Tokyo, Japan). Anterior facial photographs of all subjects on the MirS were obtained using three cameras.

The initial mirror system was proposed to save time and reduce the cost of photo-taking [9]. We adapted and enhanced the mirror system into the MirS to include basic photographic elements and photogrammetry for anthropometry (Fig. 1). The structures, mirrors, lighting, dimensions, and appendages were as follows: the frame was made of acrylic because it is light yet sturdy, can be easily cleaned, and has light-reflection properties. Five adjustable mirrors were added to the framework: two upright mirrors on the sides for lateral and oblique views, and one

\section{Fig. 1. Working concept of the MirS (without specifications)}

The facial figure is for an illustrative purpose only; it does not represent facial areas that are included in the study. The single-headed arrow indicates the built-in measuring tape to mark the distance of the camera lens from the frame. MirS, Mirror Stand.

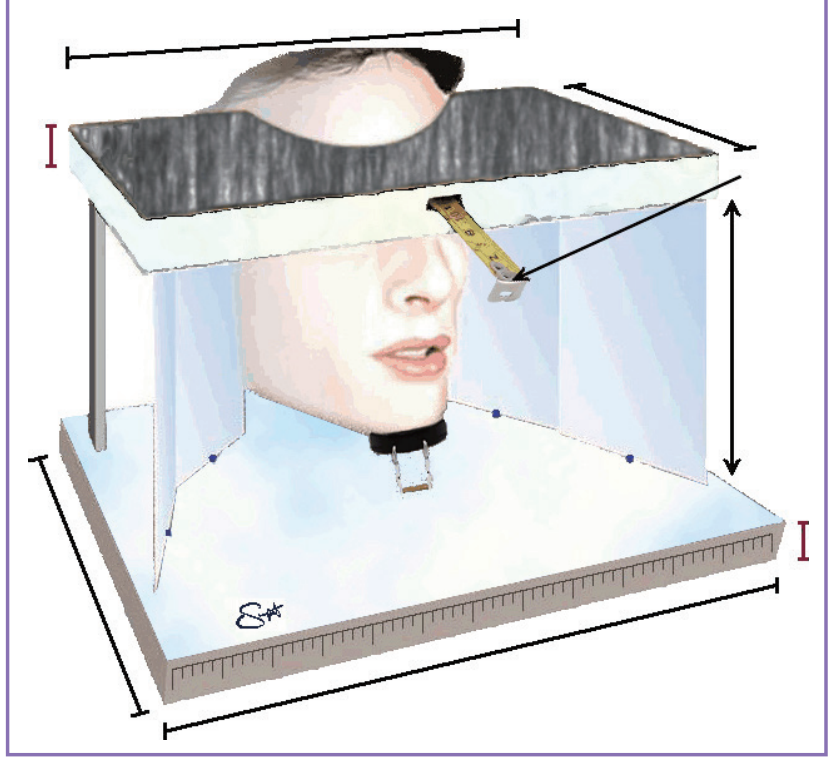

horizontal mirror midfloor for a worm's eye view. Three neon light sticks $(60 \mathrm{~W})$ were placed inside the roof, layered by a white cloth to evenly illuminate the lights. The floor was made of white matte acrylic to prevent light reflection. No background light or cover was required because the whole face of the subject would fall within the frame with no background capture. The lights were connected to an extension wire, which had to be plugged into an electrical source. This was opted for instead of portable battery packs in order to obtain a constant source of energy for providing stable light intensity. A curved chin rest was attached in the middle of the frame. The height and width of the frame and the chinrest in the middle floor could be adjusted to accommodate the differences in adult facial sizes. Measuring grids were added to serve as the intra-photograph reference scale. The floor of the side mirrors had grids to determine the equal angle of the mirrors. A measuring tape was added inside the roof construct on the front upper part of the frame to mark the distance between the camera and the frame.

The MirS was mounted onto an adjustable Mayo table, and the camera was attached to a tripod. The subjects underwent facial anthropometric markings and measurements followed by photography in one session. The photography was performed indoors with no specific requirement and in the same location for all the subjects. Fifteen anatomical landmark points were marked on each patient's face (Table 1, Fig. 2); these landmark points were adapted from Ozdemir et al. [10] 2009. Eight measurements were obtained from the 15 landmarks (Table 2) by using a straight rigid ruler. Measurements were taken in millimeters and documented in forms labeled with numbers. Each subject was seated upright with his/her chin resting against the chin rest, and the height of the device was adjusted until the head was in the Frankfort horizontal plane [11] or the natural head position. The camera height was adjusted until the camera lens was level with the subject's eyes (Fig. 3).

All photographs were digitally stored and analyzed under blinding, after the photography was completed for 40 subjects. The reliability of the three cameras was calculated using the data of five random subjects by using the intraclass correlation coefficient (ICC) test, with absolute agreement.

To begin the analysis, five subjects were selected randomly from the 40 study subjects. The ICC score of the five subjects was more than 0.99 for all the measured parameters, which showed that the three cameras produced equally reliable measurements. Thereafter, the photogrammetry analyses were carried out using one camera only (Canon EOS 600 D).

A comparison for the translatability of the eight measurement points in 40 subjects between the real facial measurements (FMs) and the photographic measurements (PMs) was carried out. 
Table 1. The ten facial anthropometric landmarks used in this study

\begin{tabular}{|c|c|c|}
\hline Landmarks & Region & Definition \\
\hline n-nasion & Face & The midpoint of the nasofrontal suture. \\
\hline mf-maxillofrontale & Nose & The anterior lacrimal crest of the maxilla at the frontomaxillary suture. \\
\hline al-alare & Nose & The most lateral point on the nasal ala. \\
\hline sn-subnasale & Face & $\begin{array}{l}\text { The junction between the lower border of the nasal septum, the partition that divides the nostrils, and the cutaneous portion of the } \\
\text { upper lip in the midline. }\end{array}$ \\
\hline cph-crista philtre & Orolabial & The point on the crest of the philtrum, the vertical groove in the median portion of the upper lip, just above the vermilion border. \\
\hline Is-labiale superius & Orolabial & The midpoint of the vermilion border of the upper lip. \\
\hline ch-cheilion & Orolabial & The outer corner of the mouth where the outer edges of the upper and lower vermilions meet. \\
\hline sto-stomion & Face & The midpoint of the labial fissure when the lips are closed naturally. \\
\hline sl-Sublabiale & Face & The midpoint of the labiomental sulcus. \\
\hline fz-Frontozygomaticus & Cranial & The most lateral point on the frontozygomatic suture. \\
\hline
\end{tabular}

\section{Fig. 2. Facial anthropometric points of interest}

The ten anthropometric points of interest marked on the subjects' face. $f z-f z$, supraorbital breadth; $m f-m f$, nasal root width; al-al, nose width; cph-cph, philtrum width; ch-ch, labial fissure width; n-sn, nose height; sn-Is, philtrum length; sto-sl, lower lip height.

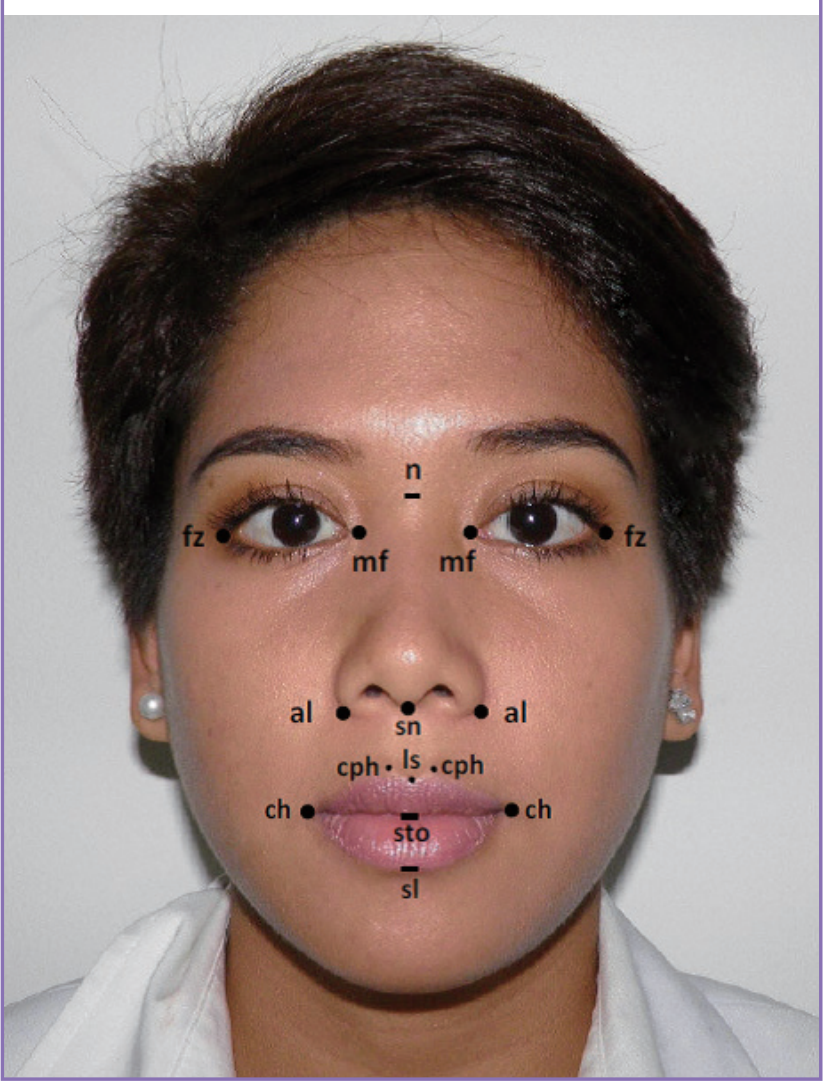

The distance between two points in the PMs was labeled 100S if the face size-in-reality and the face size-in-photo were 1 to 1 in ratio (100\% scale). For all the measured points, particularly the philtrum width (PW), very similar values were obtained from FMs versus PMs (Table 3). Disparities between FMs and PMs were statistically significant for the following five parameters:
Table 2. The eight parameters used in this study from the points drawn on the face

\begin{tabular}{|lll|}
\hline No. & Measurement name & Landmarks \\
\hline 1 & Supraorbital breadth & $\mathrm{fz}-\mathrm{fz}$ \\
2 & Nasal root width & $\mathrm{mf}-\mathrm{mf}$ \\
3 & Nose width & $\mathrm{al}-\mathrm{al}$ \\
4 & Philtrum width & $\mathrm{cph}-\mathrm{cph}$ \\
5 & Labial fissure width & $\mathrm{ch}-\mathrm{ch}$ \\
6 & Nose height & $\mathrm{n}-\mathrm{sn}$ \\
7 & Philtrum length & sn-ls \\
8 & Lower lip height & sto-sl \\
\hline Adapted from Ozdemir et al. Aesthet Plast Surg 2009;33:175-84 [10].
\end{tabular}

Fig. 3. Six photograph positions through one photo shoot

Six facial photograph positions could be obtained simultaneously with the MirS through one photo shoot; they include the anteroposterior view, right and left oblique views, right and left profile views, and the worm's eye view.

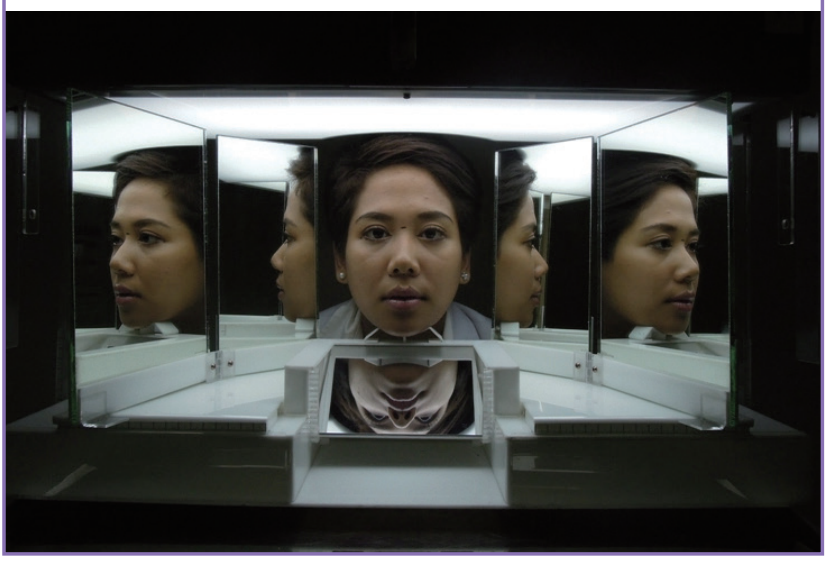

supraorbital breadth, nasal root width, nasal width, nose height $(\mathrm{NH})$, and philtrum length (PL). Bearing in mind its clinical value, the actual numeric disparity between FMs and PMs was calculated. The mean delta values represent this numeric differ- 
Table 3. Real anthropometric measurements compared with photographic measurements at "100\% scale" (100S; 1-to-1 ratio between the face in the photograph and the real face)

\begin{tabular}{|lccc|}
\hline Photographic measurements & Real values (min, max) & Photo values (min, max) & P-value \\
\hline Supraorbital breadth $(\mathrm{mm})$ & $102.0(94,117)$ & $102.8(95,116)$ & 0.014 \\
Nasal root width (mm) & $31.0(26,37)$ & $32.0(27,39)$ & 0.003 \\
Nasal width (mm) & $37.0(30,43)$ & $36.0(29,45)$ & 0.000 \\
Philtrum width (mm) & $12.8(\mathrm{SD}, \pm 2.2)$ & $12.8(\mathrm{SD}, \pm 2.1)$ & 0.323 \\
Labial fissure width (mm) & $53.0(44,65)$ & $53.0(45,63)$ & 0.728 \\
Nose height (mm) & $52.0(45,62)$ & $53.3(47,62)$ & 0.000 \\
Philtrum length (mm) & $15.0(10,21)$ & $15.3(11,20)$ & 0.030 \\
Lower lip height (mm) & $10.5(\mathrm{SD}, \pm 1.8)$ & $10.4(\mathrm{SD}, \pm 1.9)$ & 0.220 \\
\hline Min, minimum value; Max, maximum value; SD, standard deviation. & & \\
\hline
\end{tabular}

ence. The mean delta value is the lowest for PW at $0.03 \mathrm{~mm}$ (standard deviation [SD], 0.16) and the highest for $\mathrm{NH}$ at 1.66 $\mathrm{mm}(\mathrm{SD}, 1.16)$.

Further, PMs were taken once the photographs were resized to $150 \%(150 \mathrm{~S})$ and 50\% (50S) from their baseline 100\% (100S). These values were expected to replicate the actual FMs on the scale of 1.5 times and 0.5 times, respectively, to prove the consistency of the relative measurements when the photographs were resized. The measurements taken from the 150S and 50S photographs were fractioned onto the actual FMs for all eight anthropometric distances and then, averaged. The ideal expected value was 1.5 for the $150 \mathrm{~S}$ group and 0.5 for the $50 \mathrm{~S}$ group. The obtained mean value for all the measured distances was 1.51 (SD, \pm 0.07$)$ in the $150 \mathrm{~S}$ case and $0.50(\mathrm{SD}, \pm 0.03)$ in the $50 \mathrm{~S}$ case. This implied that upon photographic resizing, the values of PM are as reliable as the FM values.

The next finding, and perhaps the most important point that answered the initial question in this study, was the consistency of the photo-to-real ratio between the PMs and the real FMs. This ratio was obtained when the real ruler was lined up next to the photographic ruler scale at the desired enlargement levels, thereby enabling the conversion of photographic distances into actual distances. This constant remained steady even when the photographs were resized (in this study, to $100 \%, 150 \%$, and $50 \%)$, and the results were reproducible in all scales. At $100 \mathrm{~S}$ (equal to real face size), $1 \mathrm{~cm}$ on the photographic ruler translated to $14 \mathrm{~mm}$ in length. At $150 \mathrm{~S}, 1 \mathrm{~cm}$ on the photographic ruler translated to $21 \mathrm{~mm}(1.5 \times 100 S)$, and at $50 \mathrm{~S}$, to $7 \mathrm{~mm}(0.5 \times 100 S)$, for all the considered landmarks.

\section{DISCUSSION}

Photodocumentation in plastic surgery is vital as a clinical, communication, educational, legal, and research tool. Most clinicians obtain their own photographs without realizing that non-standard photographs have diminished analytic value. The most es- tablished standard is to either setup a studio room or use a threedimensional imaging system such as Vectra (Fairfield, NJ, USA) [12]; both are not portable and require a designated room or designated personnel. Many clinicians run a few practices and are less likely to have a photography studio or Vectra available at each practice. Therefore, a cost-effective and portable device, the MirS was developed. It aims to produce standardized photographs combined with photogrammetry to enable the translation of the produced photographs into real anthropometric measurements. At this point, the photogrammetric translation is twodimensional and is limited to the facial area.

Three cameras of different specifications were intentionally selected to prove that all cameras will produce equally reliable pictures for photogrammetry using the MirS. This is shown to be true with an intraclass correlation of more than 0.99 for all measurements between cameras. As picture quality is not of interest here, resolutions and other specifications are disregarded. Any photographs with sufficient clarity to enable the visualization of the in-photo ruler grid; the facial markings were rendered eligible. Typically, a constant distance from the subject to the camera is preferred to prevent very close or very far photographs. This is why the same camera and the same distance are set up in a studio. Since the subjects are constant with respect to the MirS, the placement and type of cameras do not have to be constant as long as the whole box is captured in the photographs.

From the eight distances measured, the delta average of the measured points is a maximum of $1.66 \mathrm{~mm}$ between the photographic and the actual measurements. This error of less than 2 $\mathrm{mm}$ may not be clinically relevant. Another explanation includes the human bias in the inter-measurement errors. Even when one examiner performs multiple distance measurements, the result may vary from one attempt to another by $1-2 \mathrm{~mm}$. Nevertheless, even when the photographs obtained on the MirS are resized, such as in this study, to $150 \%$ and $50 \%$ of the original size, the dimensions among parts of the image remain constant. The measurements of the $150 \mathrm{~S}$ group are on average 1.5 times the 
real values, while those of the $50 \mathrm{~S}$ group are on average 0.5 times the real values. Along with this constant upscaling and downscaling ratio, we also found a consistent pattern of the photoruler ratio as compared to the real-ruler values. This constant photo-to-reality ratio is the magic number in translating photographic values from the MirS into real anthropometric values.

In summary, variables that are required to produce consistent photographs with respect to measurements can be controlled using the MirS device. With the built-in accessories of the MirS, there is no need for specific light sources, backgrounds, or photography locations. The facial photographs are shown to be reproducible in terms of photogrammetry for the purpose of anthropometry. The MirS may be beneficial for use in daily practice because it is less expensive and more portable than establishing a photography studio or a three-dimensional imaging machine. It offers a portable, efficient, and cost-effective alternative for obtaining standard facial photographs of adults.

\section{REFERENCES}

1. Jemec BI, Jemec GB. Photographic surgery: standards in clinical photography. Aesthetic Plast Surg 1986;10:177-80.

2. Sommer DD, Mendelsohn M. Pitfalls of nonstandardized photography in facial plastic surgery patients. Plast Reconstr Surg 2004;114:10-4.

3. DiBernardo BE, Adams RL, Krause J, et al. Photographic standards in plastic surgery. Plast Reconstr Surg 1998;102:
559-68.

4. DiSaia JP, Ptak JJ, Achauer BM. Digital photography for the plastic surgeon. Plast Reconstr Surg 1998;102:569-73.

5. Galdino GM, Vogel JE, Vander Kolk CA. Standardizing digital photography: it's not all in the eye of the beholder. Plast Reconstr Surg 2001;108:1334-44.

6. Persichetti P, Simone P, Langella M, et al. Digital photography in plastic surgery: how to achieve reasonable standardization outside a photographic studio. Aesthetic Plast Surg 2007;31:194-200.

7. Yavuzer R, Smirnes S, Jackson IT. Guidelines for standard photography in plastic surgery. Ann Plast Surg 2001;46:293300.

8. Kraus K. Photogrammetry geometry from images and laser scans. 2nd ed. Berlin: Walter De Gruyter; 2007.

9. Kuhnel T, Wolf S. Mirror system for photodocumentation in plastic and aesthetic surgery. Br J Plast Surg 2005;58:830-2.

10. Ozdemir ST, Sigirli D, Ercan I, et al. Photographic facial soft tissue analysis of healthy Turkish young adults: anthropometric measurements. Aesthetic Plast Surg 2009;33:175-84.

11. Mosby Inc. Mosby's dictionary of medicine, nursing \& health professions. St. Louis: Mosby; 2012.

12. Metzler P, Sun Y, Zemann W, et al. Validity of the 3D VECTRA photogrammetric surface imaging system for craniomaxillofacial anthropometric measurements. Oral Maxillofac Surg 2014;18:297-304. 